\title{
SEMIPRIME FDI-RINGS
}

\author{
Nadiya Gubareni \\ Institute of Mathematics, Czestochowa University of Technology \\ Częstochowa, Poland \\ nadiya.gubareni@yahoo.pl
}

\begin{abstract}
In this paper we present some results for FDI-rings, i.e. rings with a complete set of pairwise orthogonal primitive idempotents. We consider the nilpotency index of ideals and give its upper band for ideals in some classes of rings. We also give a new proof of a criterion of semiprime FDI-rings to be prime.
\end{abstract}

Keywords: semiprime rings, prime rings, nilpotent ideal, nilpotency index, Jacobson radical, semiperfect ring, complete set of idempotents, FDI-rings

\section{Introduction}

In this paper we consider FDI-rings, i.e. rings with a finite decomposition of identity into a sum of pairwise orthogonal primitive idempotents, which form the important class of rings with finiteness conditions. We present some results for these rings.

In section 1 we consider a criterium of nilpotency for ideals and give some upper bounds for nilpotency index of nilpotent ideals in some classes in rings.

Some properties of FDI-rings connected with idempotents in them are considered in section 2 .

In section 3 we present a new proof of the theorem which yields a criterion for a semiprime FDI-ring to be decomposable into a finite direct product of prime rings. This theorem was first proved in [1] and it can be considered as a generalization of the theorem for semiprime semiperfect rings proved in [2].

The proof of the theorem for semiprime FDI-rings given here in this paper is much more similar to the proofs of structure theorems for semihereditary FDI-rings given by Drozd in [3] and for piecewise domains given by Gordon and Small in [4]. Thus, it shows the close connections between these classes of rings.

Throughout this paper all rings are associative with identity.

\section{The nilpotency index of ideals}

The notion of nilpotency is very important in the theory of rings and algebras, just as it is in other different fields of mathematics. Recall that a non-zero element 
$a$ of a ring $A$ is called nilpotent if there exists an integer $n>0$ such that $a^{n}=0$. The smallest such $n$ is called the nilpotency index of $a$. A right (or left) ideal is called a nil-ideal if its every element is nilpotent. The set $N(A)$ of all nilpotent elements of $A$ is its two-sided ideal and it is called the nilradical of $A$. A right ideal $I$ of a ring $A$ is called nilpotent if $I^{n}=0$ for some positive integer $n$. The smallest integer $n>0$ such that $I^{n}=0$ but $I^{n-1} \neq 0$ is called the nilpotency index (or index of nilpotency) of the ideal $I$ and we write it $t(I)$. It is well known that the Jacobson radical of a ring $A$ contains all one-sided nil-ideals, the prime radical of a ring $A$ is a nil-ideal and it contains all nilpotent one-sided ideals of $A$. If a ring $A$ is right Noetherian, then every one-sided nil-ideal of $A$ is nilpotent, by the theorem of Levitzki (see [5]), and the prime radical of $A$ is the largest nilpotent ideal in $A$ (see [6, Proposition 11.2.11]). Also there are well-known examples of rings containing nil-ideals that are not nilpotent.

The sum of all nil-ideals in $A$ is its two-sided ideal $N(A)$ and it is called the nilradical of $R$. It is well known that $N(A)$ is the largest nil-ideal in $A$ and there are rings for which the nilradical is not nilpotent.

Recall that an element $e$ of a ring $A$ is called an idempotent if $e^{2}=e$. If $e \in A$ is an idempotent then $e$ and $1-e$ are orthogonal idempotents such that $1=e+(1-e)$. Obviously, $e A e$ is a ring with identity $e$ for any idempotent $e \in A$. If $I$ is a two-sided ideal in $A$, then eIe is a two-sided ideal in a ring $e A e$.

In this section we will give some corollaries from the following important statement whose proof can be found in [5]:

Proposition 1.1. [5, Lemma 2.7.13]. Suppose $e$ is an idempotent of a ring $A$ and $I$ is a two-sided ideal of $A$. Then $I$ is nilpotent if and only if eIe and $(1-e) I(1-e)$ are nilpotent.

The proof of this proposition can be found in [5], but we include it for the sake of completeness and to obtain important corollaries using this proof.

Proof.

1. Let $I$ be a two-sided nilpotent ideal of $A$ with nilpotency index $t(I)=n$. Suppose that $e^{2}=e$ is an idempotent of $A$ and $x_{1}, x_{2}, \ldots, x_{n} \in e I e$. Then $x_{i}=e y_{i} e$ for some $y_{i} \in I, i=1,2, \ldots, n$, and $x_{1} x_{2} \ldots x_{n}=e y_{1} e e y_{2} e \ldots e y_{n} e=e y_{1} e y_{2} e \ldots y_{n} e=z_{1} z_{2} \ldots z_{n}=0$, where $z_{i}=e y_{i} \in e I$.

2. For the converse, suppose that $e^{2}=e$ is an idempotent of $A$ and ideals $e I e$ and $(1-e) I(1-e)$ are nilpotent with $t(e I e)=n$ and $t((1-e) I(1-e))=m$. Put $k=m+n$. Let $x_{1}, x_{2}, \ldots, x_{n} \in I$. Since $1=e+(1-e)$,

$$
\begin{aligned}
u & =x_{1} x_{2} \ldots x_{k}=[e+(1-e)] x_{1}[e+(1-e)] x_{2}[e+(1-e)] \ldots[e+(1-e)] x_{k}[e+(1-e)]= \\
& =\sum e_{i_{1}} x_{1} e_{i_{2}} x_{2} \ldots e_{i_{k}} x_{k} e_{i_{k+1}},
\end{aligned}
$$


where each idempotent $e_{i_{s}}$ is either $e$ or $1-e$. Consider an element $y=e_{i_{1}} x_{1} e_{i_{2}} x_{2} \ldots e_{i_{k}} x_{k} e_{i_{k+1}}$. Since we have $k+1>n+m$ idempotents in this product, either the number of idempotent $e$ is more than $n$ or the number of idempotent $1-e$ is more than $m$ in this product. In any case, each $y=0$, and so $u=0$. Therefore $I$ is a nilpotent ideal.

Right from the proof of Proposition 1.1 we have the following result.

Corollary 1.2. Suppose $e$ is an idempotent of a $\operatorname{ring} A$ and $I$ is a two-sided ideal of $A$ with nilpotency index $t(I)$. Then

$$
t(I) \leq n+m
$$

where $n=t(e I e)$ and $m=t[(1-e) I(1-e)]$.

Definition 1.3. A finite set of pairwise orthogonal idempotents $e_{1}, e_{2}, \ldots, e_{m}$ of a ring $A$ is called complete if

$$
e_{1}+e_{2}+\ldots+e_{m}=1 \in A \text {. }
$$

Theorem 1.4. Let $A$ be a ring with finite complete set of pairwise orthogonal idempotents $\mathrm{S}=\left\{e_{1}, e_{2}, \ldots, e_{k}\right\}$. Then a two-sided ideal $I$ of $A$ is nilpotent if and only if $e I e$ is nilpotent for any idempotent $e \in \mathrm{S}$. Moreover,

$$
t(I) \leq n_{1}+n_{2}+\ldots+n_{k} .
$$

where $n_{i}=t\left(e_{i} I e_{i}\right)$.

Proof.

Apply the induction to Proposition 1.1 and Corollary 1.2.

By Proposition 1.1, the theorem is valid if $k=2$ in the decomposition (2). Assume that theorem is true if $n \leq k-1$. Set $e=e_{1}+e_{2}+\ldots+e_{k-1}, f=1-e=e_{k}$. Then eIe and fIf are nilpotent, by Proposition 1.1, and $t(I) \leq m_{k-1}+n_{k}$, where $m_{k-1}=t(e I e)$ and $n_{k}=t(f I f)$. Since eIe is a nilpotent two-sided ideal in $e A e$, by assumption

$$
t(e I e) \leq n_{1}+n_{2}+\ldots+n_{k-1} .
$$

So, $t(I) \leq n_{1}+n_{2}+\ldots+n_{k}$.

Recall that $A$ is a semiperfect ring if any finitely generated $A$-module has a projective cover [7]. This is equivalent to the condition that the identity of $A$ can be decomposed into a finite sum of pairwise orthogonal local idempotents [8]. So that a ring $A$ is semiperfect if it has a finite complete set of local idempotents. If $e$ is a non-zero idempotent of a semiperfect ring $A$ then $e A e$ is also a semiperfect ring with the identity $e$ (see [6, Corollary 10.3.11]). Applying Theorem 2.4 to a semi- 
perfect ring we obtain the following result which is some extension of [6, Theorem 11.4.1].

Proposition 1.5. Suppose $A$ is a semiperfect ring with a finite complete set of pairwise orthogonal local idempotents $\mathrm{S}=\left\{e_{1}, e_{2}, \ldots, e_{m}\right\}$. Then a two-sided ideal $I$ in $A$ is nilpotent if and only if eIe is nilpotent for any idempotent $e \in \mathrm{S}$. Moreover,

$$
t(I) \leq n_{1}+n_{2}+\ldots+n_{k} .
$$

where $n_{i}=t\left(e_{i} I e_{i}\right)$.

In particular, if $e_{i} I e_{i}=0$ for every local idempotent $e_{i} \in A$ from the decomposition (4), then $I$ is nilpotent.

In particular, if $I=J(A)$ is the Jacobson radical of a semiperfect ring $A$, we immediately obtain the following corollary.

Corollary 1.6. The Jacobson radical $J(A)$ of a semiperfect $\operatorname{ring} A$ is nilpotent if an ideal $e_{i} J(A) e_{i}$ is nilpotent for every local idempotent $e_{i} \in A$ in some decomposition (4) of the identity of $A$ into a finite sum of pairwise orthogonal local idempotents. In particular, if $e_{i} J(A) e_{i}=0$ for every local idempotent $e_{i} \in A$ from some decomposition (4), then $J(A)$ is nilpotent.

\section{FDI-rings}

Recall that an idempotent $e^{2}=e \in A$ is called primitive if it cannot be written as a sum of two non-zero pairwise orthogonal idempotents of $A$.

Consider the following important class of rings with finiteness condition.

Definition 2.1. [6, Chapter 2]. A ring $A$ is called an FDI-ring if it has a (finite) complete set $\mathrm{S}=\left\{e_{1}, e_{2}, \ldots, e_{n}\right\}$ of orthogonal primitive idempotents, i.e. there exists a decomposition of the identity $1 \in A$ into a finite sum $1=e_{1}+\ldots+e_{n}$ of pairwise orthogonal primitive idempotents.

In this case the right (left) regular $A$-module $A_{A}\left({ }_{A} A\right)$ can be decomposed into a finite direct sum of indecomposable modules $e_{i} A\left(A e_{i}\right)$.

Note that the decomposition of $1 \in A$, given in the definition of an FDI-ring, may not be unique. Sometimes FDI-rings called rings having enough finite set of idempotents (see [4]).

Examples 2.2. The following rings are FDI-rings:

1. Division rings.

2. Finite direct sums of FDI-rings. 
3. Rings which are finite dimensional vector spaces over a division ring.

4. The ring of matrices $M_{n}(D)$ over a division ring $D$.

5. Semisimple rings.

6. Right (left) Artinian rings.

7. Right (left) Noetherian rings.

8. Semiperfect rings.

9. Right finite-dimensional rings, i.e rings which do not contain infinite direct sum of non-zero right ideals.

10. Perfect rings.

Recall the following important results:

Proposition 2.3. (See [5, Theorem 2.4.14, Corollary 2.4.15]).

(i) Let $A$ be an FDI-ring. Then the identity of $A$ can be written as a sum of a finite number of orthogonal centrally primitive idempotents.

(ii) Any FDI-ring can be uniquely decomposed into a direct product of a finite number of indecomposable rings.

Lemma 2.4. Let $A$ be an FDI-ring with finite complete set $\mathrm{S}=\left\{e_{1}, e_{2}, \ldots, e_{n}\right\}$ of orthogonal primitive idempotents. Then $c A c$ is also an FDI-ring for any idempotent $c=c_{1}+c_{2}+\ldots+c_{m}$, where each $c_{i} \in \mathrm{P} \subseteq \mathrm{S}$.

Proof.

Let $A$ be an FDI-ring with a complete set of primitive pairwise orthogonal idempotents $\mathrm{S}=\left\{e_{1}, e_{2}, \ldots, e_{n}\right\}$, i.e. there is a decomposition $1=e_{1}+e_{2}+\ldots+e_{n}$ of the identity $1 \in A$ into a sum of pairwise orthogonal primitive idempotents. Let $c=c_{1}+c_{2}+\ldots+c_{m}$, where each $c_{i} \in \mathrm{P} \subseteq \mathrm{S}$, be a non-zero idempotent of $A$.

We will show that if $c e_{i} c \neq 0$ then $c e_{i} c$ is a primitive idempotent in $c A c$. To this end it suffices to show that a ring $B=\left(c e_{i} c\right)(c A c)\left(c e_{i} c\right)$ has the only idempotent $c e_{i} c$. If $e_{i}$ does not belong to $\mathrm{P}$ then $c e_{i} c=0$. If $e_{i} \in \mathrm{P}$ then $c e_{i} c=e_{i} c=c e_{i}=e_{i}$. So that $B=\left(c e_{i} c\right)(c A \mathrm{c})\left(c e_{i} c\right)=e_{i} A e_{i}$ and $c e_{i} c=e_{i}$ is the only idempotent in $B$.

Now taking into account this lemma, we can obtain the following corollary from Theorem 1.4.

Proposition 2.5. Let $A$ be an FDI-ring with finite complete set of pairwise orthogonal primitive idempotents $\mathrm{S}=\left\{e_{1}, e_{2}, \ldots, e_{m}\right\}$. Then a two-sided ideal $I$ of $A$ is nilpotent if and only if eIe is nilpotent for any idempotent $e \in \mathrm{S}$. Moreover,

$$
t(I) \leq n_{1}+n_{2}+\ldots+n_{k}
$$

where $n_{i}=t\left(e_{i} I e_{i}\right)$.

In particular, if $e_{i} I e_{i}=0$ for every primitive idempotent $e_{i} \in \mathrm{S}$, then $I$ is nilpotent. 
Definition 2.6. An idempotent $e \in A$ is called central if $e a=a e$ for all $a \in A$.

It is easy to show that an idempotent $e \in A$ is central if and only if

$$
e A(1-e)=(1-e) A e=0,
$$

if and only if

$$
e A=A e=e A e,
$$

and so that if and only if a two-sided decomposition of $A$ has the following form:

$$
A=\left[\begin{array}{cc}
e A e & 0 \\
0 & (1-e) A(1-e)
\end{array}\right] .
$$

Definition 2.7. A central idempotent $c \in A$ is called centrally primitive if it cannot be written as a sum of two non-zero pairwise orthogonal central idempotents of $A$.

Some generalizations of a notion of a central idempotent are left (or right) semicentral idempotents introduced by Birkenmeier et al. in [9].

Definition 2.8. [9]. An idempotent $e^{2}=e \in A$ is called left (resp. right) semicentral in $A$ if $A e=e A e($ resp. $e A=e A e)$.

Obviously, any central idempotent is left and right semicentral.

An idempotent $e \in A$ is left semicentral if and only if

$$
(1-e) A e=(1-e) e A e=0,
$$

and if and only if a two-sided decomposition of $A$ has the following form:

$$
A=\left[\begin{array}{cc}
e A e & e A(1-e) \\
0 & (1-e) A(1-e)
\end{array}\right] .
$$

An idempotent $e \in A$ is left semicentral if and only if for each $x \in A$ :

$$
x e=[e+(1-e)] \text { exe }=\text { exe }+(1-e) x e=\text { exe } .
$$

So that we obtain the following lemma.

Lemma 2.9. (See [9, Lemma 1.1]) The following conditions are equivalent for an idempotent $e^{2}=e \in A$ :

(1) $A e=e A e$.

(2) $(1-e) A e=0$. 
(3) $x e=$ exe for each $x \in A$.

(4) A two-sided Peirce decomposition of $A$ has the following form:

$$
A=\left[\begin{array}{cc}
e A e & e A(1-e) \\
0 & (1-e) A(1-e)
\end{array}\right]
$$

Similar conditions hold for right semicentral idempotents which we formulate in the following lemma.

Lemma 2.10. (See [9, Lemma 1.1]) The following conditions are equivalent for an idempotent $e^{2}=e \in A$ :

(1) $e A=e A e$.

(2) $e A(1-e)=0$.

(3) $e x=$ exe for each $x \in A$.

(4) A two-sided Peirce decomposition of $A$ has the following form:

$$
A=\left[\begin{array}{cc}
e A e & 0 \\
(1-e) A e & (1-e) A(1-e)
\end{array}\right]
$$

We will use the following result which was proved in [9].

Lemma 2.11. (See [9, Lemma 2.13].) Let $c=c^{2}$ be a non-zero left (or right) semicentral idempotent of an FDI-ring $A$. Then $c A c$ is also an FDI-ring. Moreover, if $\mathrm{S}=\left\{e_{1}, e_{2}, \ldots, e_{n}\right\}$ is a complete set of primitive idempotents of $A$ then there is a subset $\mathrm{P} \subseteq \mathrm{S}$ such that

$$
\left\{c g_{i} c \mid g_{i} \in \mathrm{P}\right\}
$$

is a complete set of primitive idempotents of $c A c$. If $c \neq 1$ then the set $\mathrm{P}$ has less than $n$ elements.

From this lemma and Proposition 2.4 we immediately obtain the following corollary.

\section{Corollary 2.12.}

1. Let $A, B$ be rings and $X$ be an $A$-B-bimodule. Then the ring

$$
M=\left[\begin{array}{cc}
A & X \\
0 & B
\end{array}\right]
$$

is an FDI-ring if and only if $A$ and $B$ are both FDI-rings.

2. Any FDI-ring can be uniquely decomposed into a direct product of a finite number of indecomposable FDI-rings. 


\section{Semiprime FDI-rings}

In this section we prove a criterion for a semiprime FDI-ring to be decomposable into a finite direct product of prime rings. This theorem was considered in [7]. Here we give a new proof of this theorem without use of induction on the number of idempotents.

Recall that $\operatorname{ring} A$ is prime if the product of any two non-zero two-sided ideals $I, J$ in $A$ is not equal to zero, i.e. $I J=0$ implies that $I=0$ or $J=0$. A $\operatorname{ring} A$ is semiprime if it does not contain non-zero nilpotent ideals, i.e. $I^{n}=0$ for two-sided non-zero ideal $I$ in $A$ implies that $I=0$.

The easiest example of a prime commutative ring is a $\operatorname{ring} \mathbf{Z}$ of integers and the ring $\mathbf{Z} \oplus \mathbf{Z}$ is a semiprime commutative ring. The ring

$$
\left[\begin{array}{cc}
\mathbf{Z} & n \mathbf{Z} \\
\mathbf{Z} & \mathbf{Z}
\end{array}\right]
$$

where $n$ is a positive integer, is a prime noncommutative FDI-ring.

The ring

$$
\left[\begin{array}{cc}
\mathbf{Z} & n \mathbf{Z} \\
0 & \mathbf{Z}
\end{array}\right]
$$

is an FDI-ring which is not prime.

Recall the following important result about prime and semiprime rings.

Proposition 3.1. (See [4, Proposition 9.2.13]) If $e^{2}=e \in A$ is a non-trivial idempotent of prime (semiprime) ring $A$ then the ring $e A e$ is also prime (semiprime).

We will also use the following lemma which was proved in [1].

Lemma 3.2. (See [1, Lemma 3.3]) Let $A$ be a semiprime ring and $1=g_{1}+g_{2}$ be decomposition of $1 \in A$ into a sum of two pairwise orthogonal idempotents, $A=\left[\begin{array}{cc}A_{1} & X \\ Y & A_{2}\end{array}\right]$, where $A_{1}=g_{1} A g_{1}, A_{2}=g_{2} A g_{2}, X=g_{1} A g_{2}$, and $Y=g_{2} A g_{2}$. Let $M=\left[\begin{array}{ll}M_{11} & M_{12} \\ M_{21} & M_{22}\end{array}\right]$ be an ideal in $A$ and $M_{12} \neq 0$. Then $M_{12} M_{21} \neq 0, M_{21} \neq 0$, $M_{21} M_{12} \neq 0$. Symmetrically, if $M_{21} \neq 0$, then $M_{12} \neq 0, M_{12} M_{21} \neq 0, M_{21} M_{12} \neq 0$. In particular, if $A$ is an indecomposable ring and $Y \neq 0$, then $Y X \neq 0, X \neq 0, X Y \neq 0$. 
Lemma 3.3. Let $A$ be a semiprime ring and $1=g_{1}+g_{2}$ be a decomposition of $1 \in A$ into a sum of two pairwise orthogonal idempotents, $A=\left[\begin{array}{cc}A_{1} & X \\ Y & A_{2}\end{array}\right]$, where $A_{1}=g_{1} A g_{1}$, $A_{2}=g_{2} A g_{2}, X=g_{1} A g_{2} \neq 0$, and $Y=g_{2} A g_{2} \neq 0$.

If $I=\left[\begin{array}{cc}I_{1} & I_{12} \\ I_{21} & I_{2}\end{array}\right]$ is a two-sided ideal in $A$ with $I_{12} \neq 0$ then $I_{1} \neq 0$ and $I_{2} \neq 0$.

Proof.

If $I_{12} \neq 0$ then $I_{21} \neq 0$ as well by Lemma 3.2. Suppose that $I_{1}=0$ then $I=\left[\begin{array}{cc}0 & I_{12} \\ I_{21} & I_{2}\end{array}\right]$. Since $I$ is a two-sided ideal,

$$
\boldsymbol{I}^{2}=\left[\begin{array}{cc}
I_{12} I_{21} & I_{12} I_{2} \\
I_{2} I_{21} & I_{21} I_{12}+I_{2}^{2}
\end{array}\right] \subseteq\left[\begin{array}{cc}
0 & I_{12} \\
I_{21} & I_{2}
\end{array}\right]
$$

Therefore $I_{12} I_{21}=0$ which implies $I_{12}=I_{21}=0$ by Lemma 3.2. This contradiction shows that $I_{1} \neq 0$. Analogously one can show that $I_{2} \neq 0$.

Definition 3.4. An indecomposable projective right $A$-module $P$ of an FDI-ring $A$ will be called principal if $P \cong e_{i} A$ for some primitive idempotent $e_{i}, i=1, \ldots, n$.

Theorem 3.5. Let $A$ be a semiprime FDI-ring. Suppose that $1 \in A$ has the following decomposition into a finite sum of pairwise orthogonal primitive idempotents:

$$
1=e_{1}+e_{2}+\ldots+e_{n} .
$$

Then $A$ is a finite direct product of prime rings if and only if all rings $e_{i} A e_{i}$ are prime for all $i=1,2, \ldots, n$.

Proof.

Let $A$ be a semiprime ring, and let $1=e_{1}+e_{2}+\ldots+e_{n}$ be a decomposition of the identity of $A$ into a sum of pairwise orthogonal primitive idempotents. First suppose that $A$ is a finite direct product of prime rings. Say

$$
A=A_{1} \times A_{2} \times \ldots \times A_{s} .
$$

Let $e$ be a non-trivial primitive idempotent of $A$. Then $e A e \subset A_{i}$ for some (unique) $i$. Now $\varphi: I \rightarrow e I e$ is a product preserving a surjective map from ideals in $A_{i}$ to ideals in $e A e \subset A_{i}$. Indeed let $J$ be an ideal of $e A e$. Then $A_{i} J A_{i}$ is an ideal in $A_{i}$ and $e A_{i} J A_{i} e=J$. Now let $J, J \subset e A e$ be two non-zero ideals such that $J J^{\prime}=0$. Then $A_{i} J A_{i}$ and $A_{i} I A_{i}$ are two ideals in $A_{i}$ with product zero. So at least one of them is zero as $A_{i}$ is prime. So at least one of $J, J$ is zero. This proves that $e A e$ is prime. 
We will now prove the converse statement. Let $A$ be a semiprime FDI-ring with decomposition $1=e_{1}+e_{2}+\ldots+e_{n}$ of $1 \in A$ into a sum of primitive pairwise orthogonal idempotents.

Write $e_{i} A e_{j}=A_{i j}$ and $P_{i}=e_{i} A$. It follows from Lemma 3.2 that either $A_{i j}=A_{j i}=0$ or $A_{i j} \neq 0$ and $A_{j i} \neq 0$. This yields the possibility to introduce a relation $\approx$ on the set $\{1,2, \ldots, n\}$, setting $i \approx j$ if and only if $A_{i j} \neq 0$. In this case we write $e_{i} A \approx e_{j} A$ and the principal projective modules $P_{i}$ and $P_{j}$ will be called equivalent. We now show that $\approx$ is an equivalence relation on $\{1,2, \ldots, n\}$. Indeed, $i \approx i$ is obvious. Let $i \approx j$, i.e. $A_{i j} \neq 0$. Then from Lemma 3.2 it follows that $A_{j i} \neq 0$, i.e. $j \approx i$. Suppose now that $i \approx j$ and $j \approx k$. Then $A_{i j}, A_{j i}, A_{j k}$, and $A_{k j}$ are all non-zero. Suppose that $i$ is not equivalent to $k$, i.e. $A_{i k}=0$. By Lemma 3.2 $A_{k i}=0$ as well. Suppose $f=e_{i}+e_{j}+e_{k}$ and consider a ring $f A f$ which has the following two-sided Peirce decomposition:

$$
f A f=\left[\begin{array}{ccc}
A_{i i} & A_{i j} & 0 \\
A_{j i} & A_{j j} & A_{j k} \\
0 & A_{k j} & A_{k k}
\end{array}\right] .
$$

This implies that $A_{i j} A_{j k}=0$. Therefore $I J=\left(A_{j i} A_{i j}\right)\left(A_{j k} A_{k j}\right)=0$, where $I=A_{j i} A_{i j}$ and $J=A_{j k} A_{k j}$ are non-zero ideals in $A_{j}$. Since $A_{j}$ is a prime ring, $I=0$ or $J=0$. This contradiction shows that $i \approx k$. So that $\approx$ is an equivalence relation on $\{1,2, \ldots, n\}$.

Let $\mathrm{C}_{1}, \mathrm{C}_{2}, \ldots, \mathrm{C}_{m}$ be equivalence classes on $\{1,2, \ldots, n\}$ with regard to the relation $\approx$. Set $f_{i}=\sum_{j \in C_{i}} e_{j}$, then the $f_{1}, f_{2}, \ldots f_{m}$ form a complete set of orthogonal idempotents in $A$.

We now show that $A$ is isomorphic to a direct product of rings: $A \cong \prod_{i=1}^{m} f_{i} A f_{i}$.

Indeed, let $k \in \mathrm{C}_{i}$ and $s \in \mathrm{C}_{j}$ for $i \neq j$. This means that $k$ is not equivalent to $s$ and so $A_{k s}=A_{s k}=0$, i.e. $f_{i} A f_{j}=f_{j} A f_{i}=0$ for all $i \neq j$. Hence $A \cong \prod_{i=1}^{m} f_{i} A f_{i}$. Moreover, if $B=f_{i} A f_{i}$ then $B_{B}$ is equal to a direct sum of equivalent principal modules $P_{k}=e_{k} A$ where $k \in \mathrm{C}_{i}$.

So without loss of generality, by corollary 3.10(2), one can assume that $A$ is an indecomposable semiprime FDI-ring with decomposition $1=e_{1}+e_{2}+\ldots+e_{n}$ of $1 \in A$ into a sum of primitive pairwise orthogonal idempotents and all principal right $A$-modules $P_{i}=e_{i} A$ are equivalent. This means that $A_{j k}=e_{j} A e_{k} \neq 0$ for all $j, k=1,2, \ldots, n$.

Suppose that all the $A_{i i}$ are prime rings. We will show that $A$ is a prime ring. Let $I, J$ be a non-zero two-sided ideal in $A$ such that $I J=0$. Since $I, J$ are non-zero ideals there are indices $i, j, k, s$ such that $I_{i j} \neq 0$ and $J_{k s} \neq 0$. Let $g=e_{i}+e_{j}$ and $f=e_{k}+e_{s}$. Then $g I g$ and $f J f$ are two-sided ideals in semiprime rings $g A g$ and fAf respectively, by Proposition 3.1. Therefore by Lemma $4.2 I_{i} \neq 0$ and $J_{k} \neq 0$. Consider $M=I_{i} A_{i k} J_{k} \subseteq I J$ where $A_{i k} \neq 0$ by assumption. We will show that $M \neq 0$. 
Suppose that $L=A_{i k} J_{k}=0$. Then $A_{k i} A_{i k} J_{k}=0$. Since $A_{k i} \neq 0, S_{k}=A_{k i} A_{i k} \neq 0$ by Lemma 3.2. So that $S_{k}$ is a non-zero two-sided ideal in $A_{k}$ and $S_{k} J_{k}=0$ in a prime ring $A_{k}$ with $S_{k} \neq 0$ and $J_{k} \neq 0$. This contradiction shows that $L \neq 0$. Suppose now that $L A_{k i}=A_{i k} J_{k} A_{k i}=0$. Then $A_{k i} A_{i k} J_{k} A_{k i} A_{i k}=S_{k} J_{k} S_{k}=0$ in a prime ring $A_{k}$. So that $V_{i}=A_{i k} J_{k} A_{k i} \neq 0$ and $V_{i}$ is a two-sided ideal in $A_{i}$. Since $M=I_{i} A_{i k} J_{k}=0$ implies $M A_{k i}=I_{i} A_{i k} J_{k} A_{k i}=I_{i} V_{i}=0$ in a prime ring $A_{i}$ with $I_{i} \neq 0$ and $V_{i} \neq 0$, one obtains that $M=I_{i} A_{i k} J_{k} \neq 0$. Thus $I J \neq 0$, i.e. $A$ is a prime ring.

\section{Conclusion}

In this paper we present some results about FDI-rings, which are rings with a finite complete set of primitive orthogonal idempotents. In particular, it is given a new proof of a criterion for semiprime FDI-rings to be prime. We also consider the nilpotency index of ideals and give the upper bound of the nilpotency index, in particular for ideals in semiperfect and FDI-rings.

\section{References}

[1] Dokuchaev M.A., Gubareni N.M., Kirichenko V.V., Rings with finite decomposition of identity, Ukrain. Mat. Zh. 2011, 63(3), 319-340.

[2] Kirichenko V.V., Khibina M.A., Semiperfect semidistributive rings, [in:] Infinite Groups and Related Algebraic Structures, Acad. Nauk Ukrainy, Inst. Mat., Kiev 1993, 457-480 (in Russian).

[3] Drozd Yu.A., The structure of hereditary rings, Math. Sbornik 1980, 113(155), 1(9), 161-172 (in Russian); English translation: Math. USSR Sbornik 1982, 41(1), 139-148.

[4] Gordon R., Small L.W., Piecewise domains, Journal of Algebra 1972, 23, 553-564.

[5] Rowen L.H., Ring Theory, Vol. 1, Acad. Press, New York-Boston 1988.

[6] Hazewinkel M., Gubareni N., Kirichenko V.V., Algebras, Rings and Modules, Vol. 1, Kluwer Academic Publisher, 2004.

[7] Bass H., Finistic dimension and homological generalization of semiprimary rings, Trans. Amer. Math. Soc. 95, 1960, 466-488.

[8] Müller B., On semiperfect rings, Ill. J. Math. 1970, 14, 3, 464-467.

[9] Birkenmeier G.F., Heatherly H.E., Kim J.Y., Triangular matrix representations, Journal of Algebra 2000, 230, 558-595. 